East African Medical Journal No. 8 August 2009

FACTORS RELATED TO ATTRITION IN A COHORT STUDY OF HIV IN MALAWI

O. O. Obasanjo MBBS, MPH, PhD and N. Kumwenda, MSc, MPH, PhD, Department of Epidemiology, Johns Hopkins University Bloomberg School of Hygiene and Public Health, Baltimore MD

Request for reprints to: Dr. O. O. Obasanjo, Rm. E7138, 615 N. Wolfe Street Baltimore, MD 21205

\title{
FACTORS RELATED TO ATTRITION IN A COHORT STUDY OF HIV IN MALAWI
}

\author{
O. O. OBASANJO and N. KUMWENDA
}

\begin{abstract}
ABSTRACT

Background: Longitudinal studies face power reduction due to loss to follow up (LTFU). Bias may also arise because of differences between those who stay in the study and those who are LTFU We studied factors associated with LTFU in a cohort of HIV seronegative and sera-positive mothers in urban Malawi.

Objective: To bridge the existing gaps by examining the factors associated with attrition.

Design: Longitudinal study.

Setting: Queen Elizabeth Central Hospital (QECH) and the Kamuzu Central Hospital in Blantyre, Malawi.

Subjects: One thousand three hundred and fifty three women who attended the prenatal clinic, between October 1989 and October 1990 were recruited as part of a study to determine rates and risk factors of sero-prevalence and sera-conversion of HIV -1 among this cohort.

Results: In this cohort study, 1353 women were enrolled at delivery and 1188 (88\%) returned for the first follow-up visit at three months post-partum. Of those who returned, $177(15 \%)$ were subsequently lost during the remaining months of followup. The main predictors of $L$ TFU were younger maternal age, lower educational level of the father, HIV infection of the mother, lower birth weight of the index child and mother not being married.

Conclusions: Researchers planning studies in developing countries should consider the impact of lower education and poorer infant health on study retention in developing countries.
\end{abstract}

\section{INTRODUCTION}

Longitudinal studies face reduction in power and potential bias due loss to follow up (LTFU). By reducing the sample size and number of events, LTFU reduces the precision of estimates obtained from longitudinal studies. Bias may arise because of differences between those who stay in the study and those lost to follow-up. These differences may be associated with either known or unknown exposures or to the outcome having occurred. Different participation of individuals with given exposures could bias subsequent calculations of incidence rates and relative risks of the outcome of interest (1). To assess bias from LTFU, investigators can compare levels of known variables (usually demographic variables) between those who stay in the study and those who drop out. The belief is that if the distributions of these variables in those who stay and those who drop out are similar then loss to follow up (LTFU) is occurring randomly and bias is unlikely. Presence of a difference however does not imply a bias with respect to the outcome of interest, nor does lack of a difference guarantee the absence of bias. The best way to ensure that LTFU does not create bias or reduce precision is to minimise this loss through intensive efforts to locate each cohort member (2). It is also important that before initiating a study investigators understand factors associated with LTFU so that recruitment and follow up strategies to limit LTFU can be developed. Factors associated with LTFU in cohorts from developed countries have been described in literature, but only a few studies of LTFU in developing countries exist (3). These studies however have been limited to factors associated with return for the first follow up visit; none has studied factors associated with attrition during the study period.

With the vast majority of world wideheterosexual transmission of HIV occurring in Africa (4) many longitudinal HIV studies have been instituted in the continent. Clinical trials to prevent HIV transmission 
or progression to AIDS are also occurring in Africa. All of these African studies can beimpacted by biased LTFU. This creates a compelling need for prospective data to describe factors leading to attrition in African HIV studies, which may bias the results of these studies.

Many studies have investigated factors associated with non response and attrition. Historically, these investigations have focused more on studying the differences between responders and non-responders in surveys and cross sectional studies. In 1957, Cobb et al (5) found that respondents to a health survey in the United States tended to have a higher level of worry and a greaternumber of health worries than non-responders. Participation in theirstudy was associated with younger age, higher education, previous hospitalization and higher level of current illness. They described the effect of potential non response/LTFU bias on the results of the study. Other studies have since associated younger age, less skill in occupational function, lower education and poorerhealth status with no response to recruitment efforts $(6,7)$.

In recent years, more studies have investigated factors associated with drop-out in longitudinal studies. Dudley et al (8) found younger age and less education to be important predictors of attrition in a study of gay and bisexual HIV positive and negative men in four American cities. They postulate that education is a reliable surrogate for socioeconomic status as a predictor of attrition. They also showed that infection with HIV or a sexually transmitted disease was associated with lower participation rates in the four US cities. Iversen and Sabroe (9) found that Europeans who dropped out of their study had spent less time in employment and tended to be wage earners with poor social support. Vernon et al (10) reported similar findings even after controlling for ethnicity.

To date, few studies have been carried out in Africa to identify factors associated LTFU in cohort studies although a previous study investigated early attrition in a follow up of babies of HIV positive women in Malawi (3). The social and cultural differences between Africa and the United States and Western Europe where most studies of LTFU were conducted means that conclusions of existing L TFU studies may not be generalised to Africa. This may be particularly true for HIV studies given the differences in the natural history of HIV infection between Africa and developed countries.

With the spread of the HIV virus in Africa reaching epidemic proportions, many studies have been instituted tolearn more about the epidemiology of the disease. The investigators in these studies have used standard methods to recruit and maintain follow up. Little has been published on factors, which may cause loss to in long term African studies; in particular early versus later loss to follow up and loss to follow up due to uncontrollable life event such as moving versus dropout from other reasons. This paper is the first to investigate these issues in the context of a cohort study in an African setting; we attempt to bridge the existing gaps by examining the factors associated with attrition in two cohort studies of HIV infected women in Blantyre, Malawi.

Early versus late loss to follow-up: We divided loss to follow up into two time periods (early and late) because processes and co-factors of loss may potentially differ in these time periods. Early LTFU refers to participants in a study who participate only in the recruitment round of data collection. Late LTFUs return to at least one study visit, but then subsequently discontinue. Empirically, these two types of nonparticipants have different effects on a trial. Early LTFUs provide no information on the outcome of interest in a study, analyses of such individuals thus provide limited value. The availability of some information, including some potential outcome information on late LTFUs may make analyses of the bias they introduce possible.

Drop out due to lack of interest versus moving to a new geographical area: From a social stand point, LTFU could occur for two reasons. One reason that is completely out of the control of the study is "moving"; the participant moves out of the geographical area of the study. Alternatively, the participant can remain in the area of the study but either outright lose interest or not maintain sufficient interest relative to their life demands. To some extent this is within the control of a study with unlimited resources as further inducements could regain some of these dropouts.

\section{MATERIALS AND METHODS}

Study population: The International collaboration in AIDSResearch (ICAR) was a Johns Hopkins University, Malawi Ministry of Health (JHU-MOH) collaborative project. This was a study performed following the approval of an ethics of the Johns Hopkins University School of Public Health, and the Malawi Ministry of Health. The objective of this project, which was approved by the scientific and ethical committees in Malawi and in the USA, was to describe different aspects of HIV infection and disease.

Women who attended the prenatal clinic of the Queen Elizabeth Central Hospital (QECH) and the Kamuzu Central Hospital between October 1989 and October 1990 were recruited. This was part of a cross sectional study to determine the sero-prevalence of HIV-lamong this cohort. Women from this study were subsequently recruited at the time of delivery for a prospective study to determine risk factors of HIV transmission from HIV-1 infected mothers to their children and to determine biologic and behavioral co-factors of subsequent HIV- 1 sero-conversion in uninfected mothers.

Consecutive women attending the Queen Elizabeth Central Hospital clinic, Blantyre, for their 
first antenatal visit were interviewed by a trained nurse using a standardized questionnaire. The mothers were from urban and peri-urban Blantyre, a city with about 300,000 inhabitants in southern Malawi. The questionnaire assessed socio-demographic information (age, marital status, occupation and education of the woman and her husband, smoking and alcohol ingestion by the woman and her husband, neighbourhood of residence and electricity and water in the home), obstetric history, sexually transmitted disease (STD) history for the previous three years (gonorrhoea, syphilis, pelvic inflammatory disease (PID), genital ulceration or genital warts), and number of husbands and or sexual partners in thelast three years. Maternal weight, height, and duration of gestation were recorded at the time of this first antenatal visit.

All women were offered pelvic examinations in addition to their routine antenatal assessment. Examinations were standardised and included assessment of genital ulcerations (present or not) and vulvar vaginal warts (present or not to the unaided eye without the use of acetic acid). Vaginal fluid specimens were taken for wet-mount examination; cervical specimens were obtained for gonorrhoea culture and chlamydial fluorescent antibody. After pretest counselling, blood was tested for syphilis and HIV-1 antibodies, white blood cell count and differential. The laboratory methods used have been described elsewhere (11).

Each participant was scheduled for usual routine antenatal care and follow-UP visits every three months after delivery of the child. At each follow up visit, the mother was administered a standard questionnaire evaluating interim medical problems and sexual history. In addition, blood was collected at each visit to test for HIV -I antibodies and to repeat CD4 and CD8 T-cell counts. All infants were given a full examination including weighing with a standardized scale at birth. At 1 year and 18 months of age all infants remaining in the study were tested for HIV -1 antibodies.

Women who failed to attend scheduled visits were seen at home to ascertain the survival status of the child and invited to attend the clinic for complete evaluation. Those whocould notbelocated were tracked through neighbors. If they had moved, the neighbors were questioned as to when and why. Reasons for discontinuation were coded as not interested, forvarious reasons, moved for various reasons or death (Table 1). The date of the last visit attended was coded as the date of loss to follow up. The dates for other events such as deaths were also recorded. participants who were screened, but never returned for any follow-UP visit were considered early LTFUs and were analyzed separately from late LTFUs.

Variables: The age of participants at recruitment was categorized in to the following categories below 20 years, 20 to 24 years, 25 to 29 years and $30+$ years. This was done to allow adequate sample sizes in each age group and because marriage and child-birth occurred at different rates in these age groups. Parity (including index birth) was categorized into primigravidae, two to five children and more than five births. Widowed and divorced women were coded as single along with unmarried women, since the social influences were similar in all three groups. The maternal body mass index (BMI) in kilograms per meters squared $\left(\mathrm{kg} / \mathrm{m}^{2}\right)$ was calculated and the value of $22.1 \mathrm{~kg} / \mathrm{m}^{2}$ was used as a cut-off for mal-nourishment.

Participants answered questions about the number of years of western style education they or their husbands may have received. During analysis, participants and their husbands were divided into no education, one to six years of education and greater than six years of education.

Babies weighing below 2500 grams at birth were considered underweight. We used three definitions for sexually transmitted diseases (STDs). A history ofSTD was defined as a self reported history of gonorrhoea, pelvic inflammatory disease (PID), syphilis, genital ulceration, or genital warts. AcurrentSTD was defined as presence of vulvar or vaginal ulcerations or genital warts on examination at baseline. Laboratory STD was defined as positive cultures for gonorrhoea or trichomoniasis or positive serology for syphilis from samples obtained at baseline examination.

Outcome:Drop outs and movers were defined by period and reason (Table 1). Early dropouts and movers represent non-responders (only enrolled in study but did not return for a follow-up visit). Late drop outs and movers include responders who returned for at least one visit but subsequently discontinued participation in the study before 24 months the scheduled duration of the study. Reasons for LTFU were obtained from participants and are categorized here as follows; Dropout _ a) not interested because the husband disapproves of his wife being in the study, b) does not want the pelvic exam/blood drawing, c) not interested because she is too busy with the new baby and d) the woman is not found or difficult to trace (Table la) Moving-; a) transfer of the woman because of divorce or death of the husband / family member or b) moved, no reason given. Characteristics of early drop out and movers were compared to those of individuals who died (or whose index baby died) before the return visit and those who returned for at least one visit (persisters), which includes later drop outs and movers. Similarly, characteristics of later drop outs and movers wereeach compared to, completers and those who returned to at least one visit, but subsequently died (or whose index baby subsequently died) before the end of the study.

Because of the similarities noted between movers and dropouts during the analyses, we further 
combined these two groups in both the early and late period and determined the factors associated with this new combined group.

Statistical methods: Frequency $(2 \times 2)$ tables (odds ratios and 95\% confidence intervals (CI)) and logistic regression procedures were used to identify important characteristics that predicted early LTFU (dropout and moving) and death. Kaplan-Meier and proportional hazards models (with time from the first visit as the outcome) were used to identify important characteristics that predict late drop out, moving and death. As software for competing events was not available, we censored these models at time of competing events.

\section{RESULTS}

The characteristics of the participants are summarised in Table 1 and Figure 1. One thousand three hundred and fifty three women were screened during pregnancy and enrolled at childbirth. Most (77.9\%) of the participants in this study were below 30 years of age and had at least one previous pregnancy $(72.5 \%)$. Ninety three per cent of fathers and $86.9 \%$ of the mothers in this study had at least one year of Western style school education. The majority also had some knowledge of condoms and their proper use. Eighty four per cent of the index babies weighed more than 2500 grams and would not be considered low birth weight, although $53 \%$ of the mothers were below the mean body mass index of 22.18 during the third trimester (and would thus be considered undernourished). Only 15\% of the participants reported prior STDs, however 25\% were positive for STDs by physical examination and 58\% were positive by laboratory tests. Forty eight per cent of participants had a positive serological test for the HIV antibody on enrollment.

Table 1

Characteristics of ICAR cohort study

\begin{tabular}{lcc}
\hline Characteristic & No. & $(\%)$ \\
\hline Age (years) & & \\
$30+$ & 298 & 22.1 \\
$25-29$ & 324 & 24.0 \\
$20-24$ & 441 & 32.6 \\
$<20$ & 288 & 21.3 \\
Parity (including index birth) & \\
$6+$ & 270 & 20.0 \\
$2-5$ & 711 & 52.5 \\
1 & 372 & 27.5
\end{tabular}

Father's education (years of school)

$\begin{array}{lcc}7+ & 1090 & 82.3 \\ 1-6 & 147 & 11 . \\ \text { None missing } & 88 & 6.6 \\ \text { Mother's education (years of school) }\end{array}$

$\begin{array}{lll}7+ & 697 & 52.6 \\ 1-6 & 454 & 34.3 \\ \text { None } & 174 & 13.1\end{array}$

Marital status

$\begin{array}{lll}\text { Single } & 100 & 7.4 \\ \text { Married } & 1253 & 92.6\end{array}$

Electricity in house

$\begin{array}{lll}\text { No } & 1075 & 79.5 \\ \text { Yes } & 278 & 20.5\end{array}$

Baby weight in grams

$\begin{array}{lll}>2500 & 1137 & 84.0 \\ <2500 & 216 & 16.0\end{array}$

Maternal Body Index (Quetelets)

$\begin{array}{lll}>22.18 & 542 & 46.4 \\ <22.18 & 620 & 53.4 \\ \text { Missing } & & \\ \text { Any STD signs* } & & \\ \text { No } & 1003 & 75.0 \\ \text { Yes } & 334 & 25.0\end{array}$

*Signs include skin and mouth abnormalities, lymph nodes, abnormal vulva, ulcerations and abnormal vaginal mucosa

STD indicated by lab tests*

$\begin{array}{lll}\text { Negative } & 560 & 41.8 \\ \text { Positive } & 779 & 58.2\end{array}$

${ }^{*}$ Lab tests include gc, trich. yeast, rpr, and $\mathrm{fta}$

$\begin{array}{lll}\text { HIV status } & & \\ \text { Negative } & 694 & 51.6 \\ \text { Positive } & 652 & 48.4\end{array}$

Self reported behaviour/knowledge on any STD history*

$\begin{array}{lll}\text { Negative } & 1147 & 84.8 \\ \text { Positive } & 206 & 152\end{array}$

*History includes syphilis, gonorrhoea, buboes, pelvic exam pain, herpes and warts

Knowledge of condoms

$\begin{array}{lll}\text { Negative } & 1074 & 79.9 \\ \text { Positive } & 271 & 20.1 \\ \text { Use any condoms } & & \\ \text { Negative } & 170 & 64.2 \\ \text { Positive } & 95 & 35.8\end{array}$




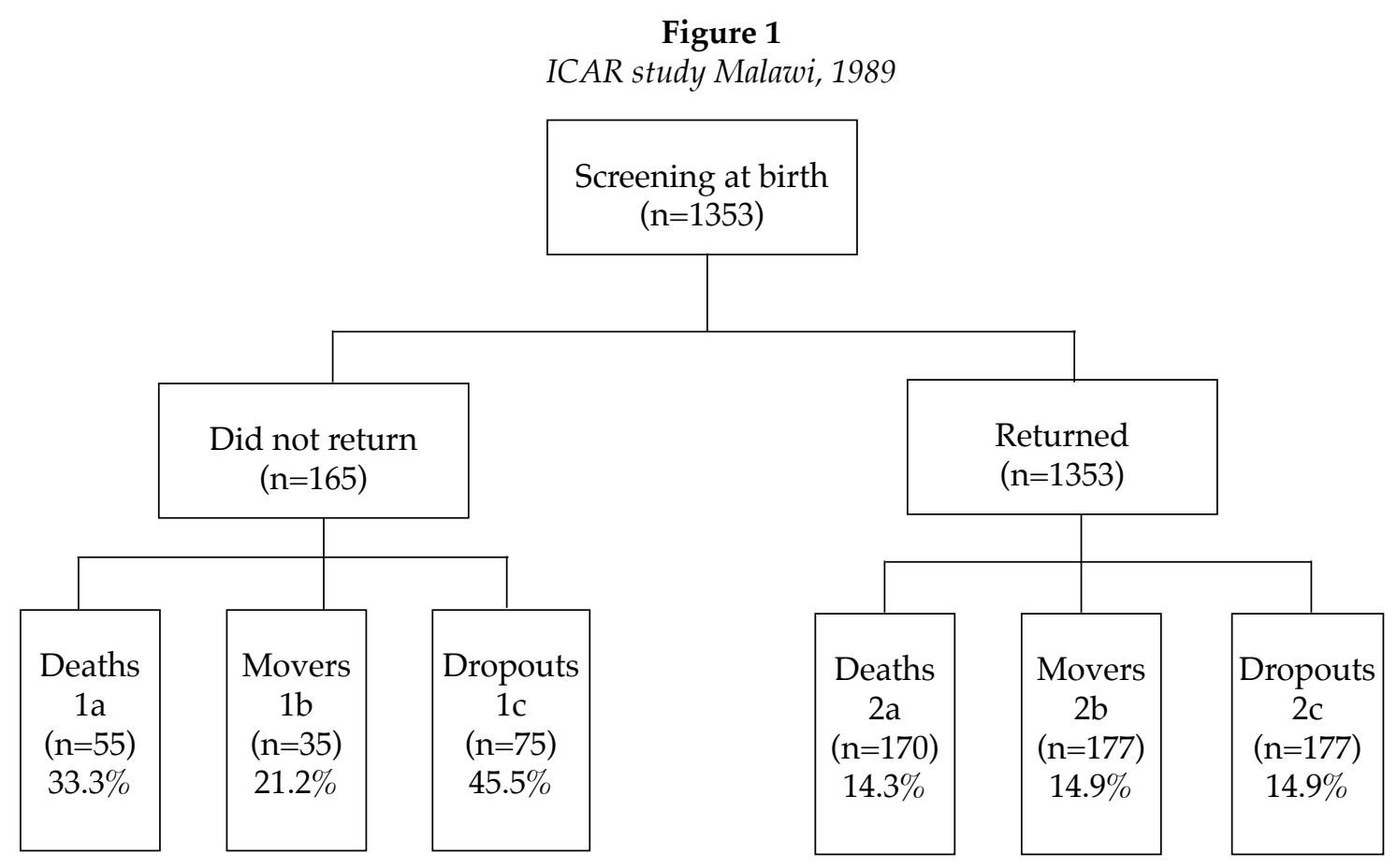

Incidence of dropout, moving and death: Table 2 presents a break down of rates of early / late dropout, moving and death and specific reasons for early / late dropout and moving. One hundred and sixty five of the participants $(12.2 \%)$ did not return for at least one follow-up visit, $55(33.3 \%)$ because of death of the index baby or mother. Thirty five $(21.2 \%)$ of the rest of these were classified as movers. The rest (75 or $41.5 \%$ ) were considered early lost to follow up according to the criteria defined earlier. Of the 1188 that returned 524 did not complete 24 months of follow-up while $664 \mathrm{did}$. Of the 524 that did not complete 24 months, 170 (31.4\%) did not because of deaths of either the index baby or mother, and 177 $(33.8 \%)$ did not complete because of uncontrollable life events such as transfer of the husband. The rest (177) were considered late drop outs. Therefore, $14.9 \%$ of the participants who returned for at least one visit were lost to follow up during the study period. The total rate of attrition among the women enrolled in the study was $44.2 \%$ at 24 months $([170+177+177] / 1188)$. The adjusted (Kaplan-Meier) rates were $10.3 \%$ at one year and $14.5 \%$ at 24 months of follow up.

Table 2a

Reasons for discontinuation of participation given for early and late period

Reason Early (N) Late $(\mathrm{N})$ Movers Drop outs Movers Drop outs

*Not found or difficult to trace

*Not interested (no reason given)

*Not interested (too busy with new baby)

*Not interested (doesn't want pelvic exam or blood drawing)

*Not interested (husband disapproves)

Moved (no location given or transfer to another city)

Moved home (no reason given)

Moved home (because of divorce/separation)

Moved home (because of death of husband or other family member) 1

*This is included as one of the definitions of loss to follow up (LTFU)

$44 \quad 60$

$27 \quad 102$

3

$14 \quad 85$

$19-63$

123


Table $2 \mathbf{b}$

Cohort ( $n=1355$ ) of early and late LTFU

\begin{tabular}{llll}
\hline Group & No. & $(\%)$ & $\begin{array}{l}\text { Mean } \\
\text { FUT(month)* }\end{array}$ \\
\hline $\begin{array}{l}\text { Early }(\mathrm{n}=1353) \\
\quad \text { Death }\end{array}$ & 55 & 4.1 & \\
$\quad$ Movers & 35 & 2.6 & \\
LTFU & 75 & 5.5 & \\
Returned to & & & \\
a Visit & 1188 & 87.8 & \\
Late (n=1188) & & & \\
$\quad$ Death & 170 & 14.3 & 9.7 \\
$\quad$ Movers & 177 & 14.9 & 7.8 \\
LTFU & 177 & 14.9 & 7.1 \\
Complete & 664 & 55.8 & 38.5 \\
\hline
\end{tabular}

${ }^{*}$ FUT=Follow-up Time=Last visit-First visit
Predictors of dropout, moving and combined LTFU: The results of the univariate and multivariate logistic model to determine predictors of early movers, early drop out, combined group (movers and dropouts) and death; each versus returnees to at least one visit are summarized in table 3. The statistically significant predictors of early drop out in the logistic model were illiteracy of the father, OR 3.71 (95\% Cl1.82-7.30), illiteracy of the mother, OR 2.18 (95\% Cll.11-4.11) and presence of electricity in the household, OR 0.46 (95\% CI 0.20-0.93). The only significant predictor of moving was a decrease in parity. The odds ratio for moving was 3.62 (95\% CI 1.04-22.88) in the univariate and multivariate analyses when those with one child were compared to those with 6 or more children. The odds ratio for those with two to five children versus $6+$ children was $4.73(95 \%$ Cll.29-30.43) for univariate and multivariate analyses.

Table 3

Logistic model predictors of early period (comparison group is those who return for at least one visit)

\begin{tabular}{|c|c|c|c|c|}
\hline \multirow[t]{2}{*}{ Characteristic } & \multicolumn{2}{|c|}{ Univariate* } & \multicolumn{2}{|c|}{ Multivariate } \\
\hline & OR & $9 \mathrm{~S} \% \mathrm{CI}$ & OR & $95 \% \mathrm{CI}$ \\
\hline \multicolumn{5}{|l|}{ Drop out } \\
\hline \multicolumn{5}{|l|}{ Father's education } \\
\hline $7+$ & 1.0 & & 1.0 & \\
\hline $1-6$ & 1.47 & $0.68-2.82$ & 1.24 & $0.57-2.43$ \\
\hline None & 3.09 & $1.47-5.98$ & 2.58 & $1.20-5.18$ \\
\hline \multicolumn{5}{|l|}{ Mother's education } \\
\hline $7+$ & 1.0 & & 1.0 & \\
\hline $1-6$ & 1.7 & $1.01-2.87$ & 1.48 & $0.87-2.54$ \\
\hline None & 2.16 & $1.11-4.11$ & 1.59 & $0.77-3.13$ \\
\hline \multicolumn{5}{|l|}{ Electricity in house } \\
\hline No & 1.0 & & 1.0 & \\
\hline Yes & 0.43 & $0.19-0.87$ & 0.49 & $0.21-0.99$ \\
\hline \multicolumn{5}{|l|}{ Movers } \\
\hline \multicolumn{5}{|l|}{ Parity } \\
\hline $6+$ & 1.0 & & 1.0 & \\
\hline $2-5$ & 4.73 & $1.29-30.43$ & 4.73 & $1.29-30.43$ \\
\hline 1 & 3.62 & $1.04-22.88$ & 3.62 & $1.04-22.88$ \\
\hline \multicolumn{5}{|c|}{ Drop out and movers } \\
\hline \multicolumn{5}{|c|}{ Father's education years } \\
\hline $7+$ & 1.0 & & 1.0 & \\
\hline $1-6$ & 1.47 & $0.77-2.61$ & 1.30 & $0.67-2.33$ \\
\hline None & 2.84 & $1.47-5.16$ & 2.41 & $1.21-4.51$ \\
\hline \multicolumn{5}{|c|}{ Mother's education years } \\
\hline $7+$ & 1.0 & & 1.0 & \\
\hline $1-6$ & 1.33 & $0.86-2.06$ & 1.33 & $0.84-2.10$ \\
\hline None & 1.85 & $1.06-3.14$ & 1.68 & $0.92-2.97$ \\
\hline
\end{tabular}




$$
\begin{aligned}
& \text { Death } \\
& \text { HIV status } \\
& \text { Negative } \quad 1.0 \quad 1.0 \\
& \begin{array}{lllll}
\text { Positive } & 3.24 & 1.78-6.21 & 2.14 & 1.14-4.21
\end{array} \\
& \text { Baby weight (grams) } \\
& >2500 \quad 1.0 \\
& \begin{array}{lllll}
<2500 & 11.84 & 6.71-21.54 & 10.12 & 5.67-18.59
\end{array}
\end{aligned}
$$

*The following variables were also examined for the drop out group but were not significant:

HIV status, parity, baby weight, age, marital, any lab tests. Any STD signs, any STD history, and maternal body mass index. (since weight and height were determined at the first follow-up visit postnatally, body mass index was not measured i.e. these women never came back after enrollment). The Mover group included only one significant variable

The Death group variables also not significant were the same as above except for HIV status and baby weight.

Illiteracy of the mother and father were independently significant predictors of the combined early LTFU outcome of either moving or dropping out. The odds ratio in fathers with noeducation was 2.84 (95\% CI1.475.16) for univariate analysis and 2.41 (95\% Cl1.21-4.51) for multivariate analysis when they were compared to those with the highest level of education.

The odds ratio for mothers with no education was $1.85(95 \% \mathrm{Cl} 1.06-3.14)$ for univariate analysis and 1.68 (95\% CI 0.92-2.97) for multivariate analysis when they were compared to those with the highest level of education.

The results of the multivariate proportional hazards model to determine predictors of late drop out are summarised in Table 4 . The statistically significant predictors of late drop out were illiteracy in the father relative hazard (RH) 1.91 (95\% CI 1.1 $0-3.31)$, the marital status of the mother, $\mathrm{RH} 2.43$ (95\% CI 1.08-5.48) and the mothers knowledge of condoms based on questionnaires $0.58(0.38-0.89)$. The significant predictor of late moving in this group was illiteracy of the father RH 2.04 (95\% CI 1.22-3.42). For combined late L TFU (moving and dropout together), the significant predictors in multivariate modes were;

\begin{tabular}{|c|c|c|c|c|}
\hline \multirow[b]{2}{*}{ Characteristic } & \multicolumn{2}{|c|}{ Univariate* } & \multicolumn{2}{|c|}{ Multivariate } \\
\hline & $\mathrm{RH}$ & $\mathrm{CI}$ & RH & CI \\
\hline \multicolumn{5}{|l|}{ Drop out } \\
\hline \multicolumn{5}{|c|}{ Father's education } \\
\hline $7+$ & 1.0 & & 1.0 & \\
\hline $1-6$ & 1.34 & $0.87-2.07$ & 1.26 & $0.81-1.94$ \\
\hline None & 1.91 & $1.10-3.31$ & 1.78 & $1.02-3.08$ \\
\hline \multicolumn{5}{|c|}{ Knowledge of condom } \\
\hline No & 1.0 & & 1.0 & \\
\hline Yes & 0.59 & $0.39-0.90$ & 0.61 & $0.40-0.93$ \\
\hline \multicolumn{5}{|l|}{ Marital } \\
\hline Single & 1.0 & & 1.0 & \\
\hline Married & 2.40 & $1.04-5.32$ & 3.30 & $1.26-8.58$ \\
\hline \multicolumn{5}{|l|}{ Movers } \\
\hline \multicolumn{5}{|c|}{ Father's education } \\
\hline $7+$ & 1.0 & & 1.0 & \\
\hline $1-6$ & 0.68 & $0.39-1.20$ & 0.68 & $0.39-120$ \\
\hline None & 2.04 & $122-3.42$ & 2.04 & $1.22-3.42$ \\
\hline
\end{tabular}
illiteracy in the father RH 1.98 (95\% CT), illiteracy in the mother RH 1.38 (95\% CI), maternal ignorance of condoms RH $0.71(95 \% \mathrm{CI})$ and having a married mother RH 1.87 (95\% CI).

Table 4

Proportional hazards predictors of hazard for late period drop out moving and death 


\begin{tabular}{|c|c|c|c|c|}
\hline \multicolumn{5}{|c|}{ Drop out and movers } \\
\hline \multicolumn{5}{|c|}{ Father's education } \\
\hline $7+$ & 1.0 & & 1.0 & \\
\hline $1-6$ & 1.00 & $0.71-1.41$ & 0.93 & $0.65-132$ \\
\hline None & 1.98 & $1.36-2.89$ & 1.73 & $1.16-257$ \\
\hline \multicolumn{5}{|c|}{ Mother's Education } \\
\hline $7+$ & 1.0 & & 1.0 & \\
\hline $1-6$ & 1.13 & $0.89-1.42$ & 1.16 & $0.85-1.61$ \\
\hline None & 1.38 & $1.03-1.88$ & 1.03 & $0.81-1.31$ \\
\hline \multicolumn{5}{|c|}{ Knowledge of condom } \\
\hline No & 1.0 & & 1.0 & \\
\hline Yes & 0.71 & $0.53-0.94$ & 0.73 & 0.54-097 \\
\hline \multicolumn{5}{|l|}{ Marital } \\
\hline Single & 1.0 & & 1.0 & \\
\hline Married & 1.87 & $1.12-3.13$ & 2.37 & $1.30-4.35$ \\
\hline \multicolumn{5}{|l|}{ Death } \\
\hline \multicolumn{5}{|c|}{ Maternal body mass index } \\
\hline$>22.16$ & 1.0 & & 1.0 & \\
\hline$<22.16$ & 1.54 & $1.12-2.12$ & 1.42 & $1.03-1.96$ \\
\hline \multicolumn{5}{|l|}{ HIV status } \\
\hline Negative & 1.0 & & 1.0 & \\
\hline Positive & 324 & $2.40-4.39$ & 2.65 & $1.95-3.59$ \\
\hline Any STD si & & & 1.0 & \\
\hline No & 1.0 & & & \\
\hline Yes & 1.80 & $1.37-2.36$ & 1.60 & $1.20-2.08$ \\
\hline Any STD hi & & & 1.0 & \\
\hline Negative & 1.0 & & & \\
\hline Positive & 1.73 & $127-2.26$ & 1.41 & $0.98-2.04$ \\
\hline
\end{tabular}

*The following variables were also examined for the drop out and moving groups but were not significant: HIV status, parity, maternal body mass index.. Baby weight, age, electricity, any lab tests, any STD signs, any STD history, and mothers education. The Death group variables also not significant were the same as above except for Maternal Body Mass Index. HIV status, and any STD signs and any STD history

Predictors of baby death: Maternal HIV status was a predictor of death (versus returning to at least one visit) in both logistic (early baby death) and proportional hazards (late baby death) models (censoring at LTFU). Both mother being $\mathrm{HIV}+, \mathrm{OR}$ 3.24 (95\% $\mathrm{Cl} 1.78-6.21)$ and baby weight $<2500 \mathrm{gm}$, OR 11.84 (95\% C16.71 - 21.54) were dramatically associated with early baby death. The birth weight of the index child (less than $2500 \mathrm{~g}$ ) was a significant predictor of late death in the proportional hazards model, RH 2.09 (95\% CI 1.43-3.04). Other predictors of late death in the proportional hazards model were maternal BMI (less than 22.18), RH 1.42 (95\% Cl 1.031.96) and presence of STDs by physical examination RH 1.60 (95\% CI 1.20-2.08). A history of signs, symptoms or treatment for an STD was significant in the univariate proportional hazards RH 1.41 (95\% CI 0.98-2.04) model for late death but dropped out in the multivariate model.

\section{DISCUSSION}

This study is the first to report factors related to attrition in a longitudinal African study. Ioannides et al (3) performed studied early dropout in a cohortin Malawi but they limited their analyses to participants who did not return for the first follow up visit (early LTFU by our definition). To our study, weinvestigated the factors that were significantly related to early and late drop prospectively during the course of the study. 
One of the most significant findings here is that lack of education of the mother and/or father predicted discontinuationbecause of moving (an uncontrollable life event) and dropout (i.e., discontinuation from lack of sufficient interest) up are similar. Illiteracy predicted both early LTFU (failing to return to at least one study visit) and late (after returning to at least one study visit) drop-out. These findings are also consonant with those of Ioannidis et al (3) who found that lower maternal and paternal education was a significant predictor of drop out. We believe education is a reliable surrogate for socioeconomic status and postulate that these poorer women are more likely to move due to economic instability, their livelihoods as well as those of their husbands are more likely to lead to leaving the study area. These less educated and poorer women are also more likely to develop life stresses such as becoming widowed or loosing a child, both associated with attrition. Another indicator of SES, the presence of electricity in the participants house was significantly associated with reduced levels of early dropout, but not with rates of early moving or of early/late dropout.

As lower education predicted both moving and drop out, this raises the possibility that the difference between our classification of a person as a "movers or a drop outs" may be an observation bias associated with the timing of the follow up visit. If the follow up visit occurred close to the period of upheaval and moving then it is possible to trace the participant and determine if she has moved and why. If the follow up visit occurs a long time following movement, then finding the cause of attrition from the study may be more difficult and the participant may be coded as drop out. We were unable to investigate this relationship because of how our data was collected. Further validating our hypothesis about SES and attrition is the finding that women who are knowledgeable about condoms tend to stay in the study (knowledge of condoms was associated with lower rates of late dropout and late combined LTFU. Women aware of condoms will probably be educated with a higher SES. Alternatively, knowledge of condoms may reflect greater awareness of HIV consequences providing greater incentive for the woman to remain in the study.

We found that low parity was associated with early moving (but not with early drop out or late moving/drop out). This may reflect Type 1 error given the number of comparisons that have been made in this study. However, it may be easier to move with one child than to move with six or more. Also, a family with many children will tend to have more social and economic ties to that community than if they had just one child, it would thus be more difficult to move. Being married was associated with late drop out and combined LTFU; again possibly a Type 1 error. When taken alone, this may seem counter intuitive, but when considered along with the fact that attrition is also associated with low SES, then the situation becomes clearer. These women are married and poor as against unmarried women. An unmarried woman even at times of social and economic difficulty may be less likely to move because situation will probably be the same or worse wherever she moves to, especially if she has children. She is better served to stay where she is and utilize whatever social support she may have. This reasoning may explain this finding.

We found that mother being HIV+, low baby birth weight; low maternal BMI and maternal STD were associated with death of the baby. All of these causes should biologically prognosticate poor health of the baby and were not associated with dropout or moving. None of the factors associated with dropout and moving (i.e., illiteracy) were associated with death of the baby. This increases our confidence in our findings confirming that those who were classified as LTFU were not those who had died and were misclassified.

Our study included large numbers of HIV positive women at the beginning of the study, since the primary outcome of this study was to determine HIV prevalence and incidence. This unfortunately represents the situation of HIV infection in that region (12). We believe these results would be generalizable to non-HIV infected subject in Malawi. As noted earlier, another limitation of this study may be difficulty ascertaining reason for dropout.

We believe our findings have importance for investigators in Africa. With the resources spent in the development of vaccines for HIV infection and malaria, it becomes imperative that participants involved in studies of the efficacy of these vaccines (or other long term treatments) stay until completion of the study. Our findings suggest that lower SES among participants may cause unacceptable attrition in these studies. Similar findings of SES markers as predictors of attrition were also recently reported in a clinical trial in neighbouring Zambia (13). Karcher et al (14) also found that lower SES as indicated by lower education was associated with LTFU in an HIV treatment cohort in Kenya. We thus suggest that researchers carrying out studies in developing countries develop quality control measures aimed at maintaining continued participation among subjects at various SES strata. Time should be spent identifying socioeconomic divisions and subdivisions, markers that identify individuals in these divisions and activities to ensure their full participation. Our findings also suggest that similar factors are responsible for attrition in the early and late periods, so researchers can develop similar methods to ensure participation during both periods. 


\section{ACKNOWLEDGEMENTS}

Diane Markakis performed all the statistical analyses. Drs. Don Hoover and Taha Taha were very supportive throughout the process of development of the idea of the study and writing of the manuscript. The authors declare that they have no competing interests

\section{REFERENCES}

1. Rothman, I.K. and Greent and, S. Modern epidemiotogy. 2nd ed. Philadelphia: Lippincott Williams and Wilkins, 1998: 6:

2. Criqui, M.H., Barret-Connor, E. and Austin, M. Differences in respondents and non-respondents in a population based cardiovascular disease study. $A m$. J. Epidemiol. 1978; 108:367-372.

3. Ioannides, I.P.A., Taha, T.E., Kumwenda, N. et al. Predictors and impact of losses to follow-up in an HIV -I perinatal transmission cohort in Malawi. Int. J. Epidemiol. 1999; 28: 769-775.

4. Ford, B. J. Aids and Africa. Biologist (London). 2000;47: 224.

5. Cobb, S., King, S. and Chew, E. Differences in respondents and non-respondents in a morbidity survey involving medical examination. J. Chron. Dis. 1957; 6: 95-108.

6. Walker, M., Shaper, A.G. and Cook, D.G. Nonparticipation and mortality in a prospective study of cardiovascular disease. J. Epidemiol. Comm. Health. 1987;41:295-299.

7. Ohlson, C. and Ydreborg, B. Participants and nonparticipants of different categories in a health surveyA cross-sectional register study. Scand. J. Soc. Med. 1985; 13: 67-74.

8. Dudley, I., Jin, S., Hoover, D. et al. The multi-center AIDS cohort study: Retention after 9 and a half years. Am. J. Epidemiol. 1995;142: 323-330.

9. Iversen, L. and Sabroe, S. Participation in a follow-up study of health among unemployed and employed people after a company close down: Drop-outs and selection bias. J. Epidemiol. Comm. Hlth. 988; 42: 396-401.

10. Vernon, S.W., Roberts, E.R. and Lee, E.S. EthnicStatus and participation in longitudinal health surveys. Am. J. Epidemiol. 1984; 119: 99-112.

11. Taha, T.E., Canner, J.K., Wangel, A. M., et al. Research on Human Immunodeficiency Virus in Malawi: The Johns Hopkins University-Ministry of Health (IHUMOH) Project. Malawi Med. J. 1994.

12. Kachapila, L. The HIV/ AIDS epidemic in Malawi. Int. Nurs. Rev. 1998; 45:179-181.

13. Kempf, M.C., Allen, S., Zulu, I., et al. Rwanda Zambia HIV Research Group. Enrollment and retention of HIV discordant couples in Lusaka, Zambia. J. Acquir. Immune. Defic. Syndr. 2008; 47: 116-125.

14. Karcher, H., Omondi, A., Odera, J., Kunz, A. and Harms, G. Risk factors for treatment denial and loss to follow-up in an antiretroviral treatment cohort in Kenya. Trop. Med. Int. Hlth. 2007; 12: 687-694. 